\title{
Visualisation of Range Shortening in Carbon Ion Beams and Washout of Positron Emitter: First-in-Human Report
}

\author{
SHINTARO SHIBA ${ }^{1,2}$, MAKOTO SAKAI $^{2}$, MASAHIKO OKAMOTO $^{1}$ and TATSUYA OHNO ${ }^{1}$ \\ ${ }^{1}$ Department of Radiation Oncology, Gunma University Graduate School of Medicine, Maebashi, Japan; \\ ${ }^{2}$ Gunma University Heavy Ion Medical Center, Maebashi, Japan
}

\begin{abstract}
Background/Aim: Auto-activation positron emission tomography (AAPET) is one of the most promising methods to verify beam range in carbon ion radiotherapy $(C$-ion $R T)$. We aimed to confirm this for the first time in a clinical setting by performing AAPET in a patient with pancreatic cancer previously receiving coil embolisation of hepatic artery pseudoaneurysm. Materials and Methods: A 70-year-old pancreatic head cancer patient was treated with $C$-ion $R T$ on a clinical dose of $4.6 \mathrm{~Gy}$ (RBE), followed by AAPET, to verify beam ranges in $C$-ion $R T$. Results: Positron emission tomography (PET) revealed low positron emitter concentrations at the distal side of the internal metals and in the aorta compared to the dose distribution of the treatment plan, indicating range shortening by internal metals in $C$-ion beams and positron emitter transportation by biofluids. Conclusion: AAPET may detect range shortening by internal metals in the trajectory and washout of intravascular positron emitter compared to plan dose distribution.
\end{abstract}

Carbon ion radiotherapy (C-ion RT) has been used for treating various cancers, with reported favourable clinical outcomes, due to its biological and physical advantages (15). Biologically, it has a higher cell-killing effect, even in radioresistant tumours, additionally its physical properties enable a dose distribution better than X-ray RT (6). Although $\mathrm{C}$-ion RT can provide higher target dose localisation, C-ion beams are particularly sensitive to the calculation accuracy since the range of $\mathrm{C}$-ion beams depends on the tissue density along the beam path (7-10). Furthermore, the presence or

This article is freely accessible online.

Correspondence to: Makoto Sakai, Gunma University Heavy Ion Medical Center, 3-39-22, Showa-machi, Maebashi, Gunma, 3718511, Japan. Tel: +81 272208378, Fax: +81 272208379, e-mail: sakai-m@gunma-u.ac.jp

Key Words: Carbon-ion radiotherapy, auto-activation positron emission tomography, irradiated site visualisation. absence of metal objects on the beam path can cause severe dose distribution variation. Therefore, verification of the beam range in $\mathrm{C}$-ion $\mathrm{RT}$ is necessary.

In terms of range estimation, the usefulness of images relies on the detection of annihilation gamma rays (pair of $511 \mathrm{keV}$ photons) using positron emission tomography (PET) and Compton camera after C-ion RT (11-14). Positron emitters (e.g., ${ }^{11} \mathrm{C},{ }^{13} \mathrm{~N}$, and ${ }^{15} \mathrm{O}$ ) are generated from nuclear interactions between incoming $\mathrm{C}$-ions and target materials, and through annihilation of a positron with an electron, annihilation gammarays are produced. Thus, the distribution of the positron emitter imaged by the PET or Compton camera correlates with the Cion trajectory. Some previous studies have reported that the distribution of annihilation gamma rays expresses the irradiated area; however, there are no imaging reports observing range shortening of $\mathrm{C}$-ion beams caused by their passing through internal metals in humans. Additionally, although transport of positron emitters via biofluids has been previously reported (12, 15-17), to the best of our knowledge, observation of washout in human blood vessels has not been reported. In this study, we verified whether these events can be observed using autoactivation PET (AAPET) in an actual treatment setting.

\section{Materials and Methods}

Patient and carbon-ion radiotherapy. A 70-year-old Japanese male patient with pancreatic head cancer (clinical T2N0M0 Stage IB based on the eighth edition of the Union for International Cancer Control/American Joint Committee on Cancer TNM staging system) received C-ion RT at Gunma University Heavy Ion Medical Center (GHMC) (Figure 1A, B, and C) (18). Five years prior to the pancreatic head cancer diagnosis, the patient had undergone an extended left hepatic lobectomy and extrahepatic bile duct resection for hilar cholangiocarcinoma. During that period, he developed a postoperative pancreatic fistula and pseudoaneurysm, and underwent coil embolisation of the hepatic artery. An informed consent was obtained from the patient before initiation of C-ion RT.

A heavy-ion accelerator at the GHMC was used to generate C-ion beams. Beam energies of $290 \mathrm{MeV} / \mathrm{u}$ in the vertical beam and 380 $\mathrm{MeV} / \mathrm{u}$ in the horizontal beam were selected according to the depth of the tumour. Doses of C-ion RT were expressed as relative biological effectiveness (RBE) weighted dose [Gy (RBE)], which 


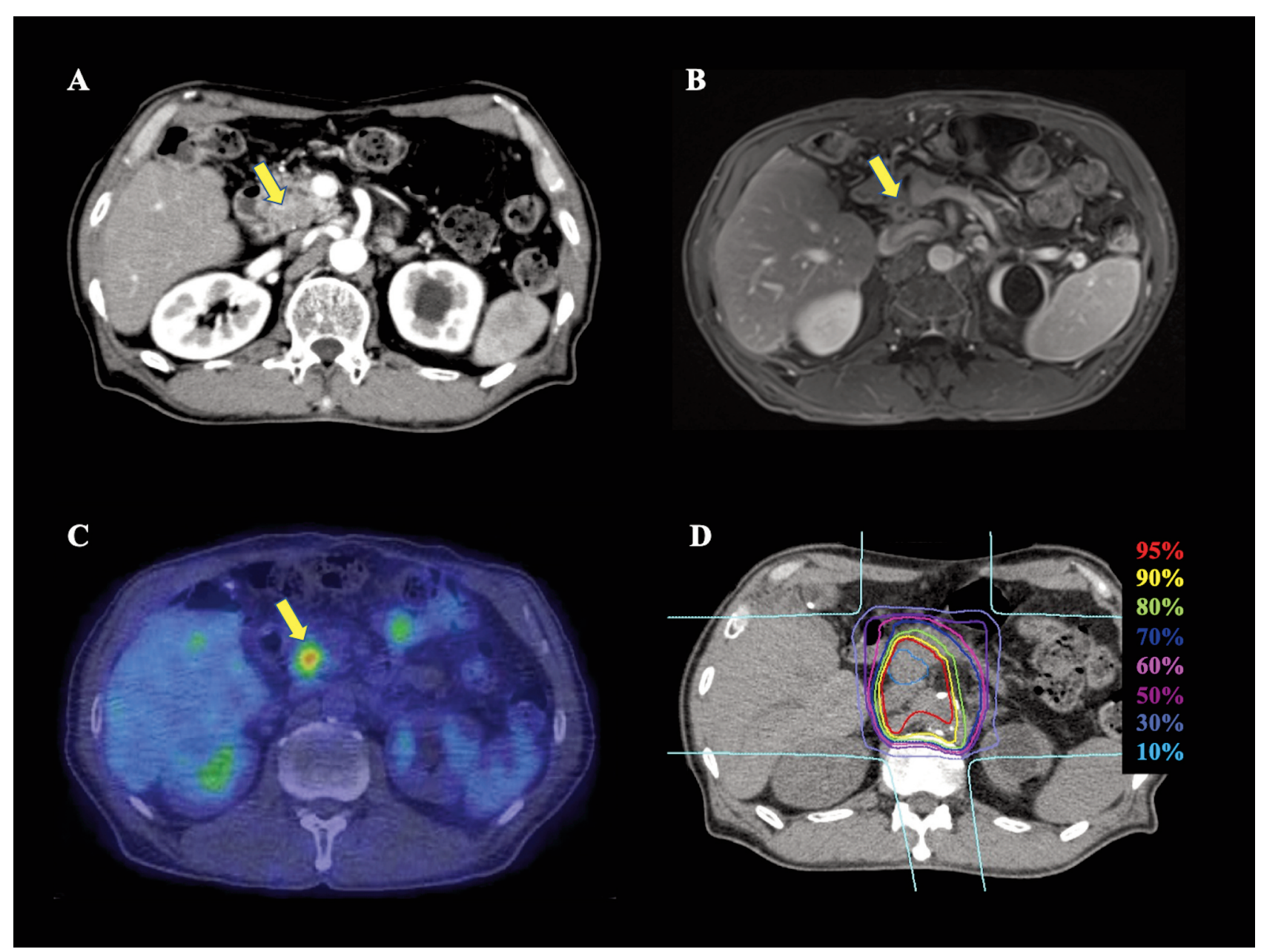

Figure 1. Radiological images before carbon ion radiotherapy (C-ion RT) and dose distribution. (A) Contrast-enhanced computed tomography (CT). The yellow arrow shows the tumour. (B) Gadolinium-enhanced magnetic resonance imaging. The yellow arrow shows the tumour. (C) 2-deoxy-2[18F]fluoro-D-glucose (FDG)-positron emission tomography (PET)/CT. The yellow arrow shows the tumour with an abnormal FDG uptake. (D) Dose distribution on axial CT images. The area within the blue outline is the pancreatic head tumour. Highlighted are the 95\% (red), 90\% (yellow), $80 \%$ (green), $70 \%$ (deep blue), 60\% (magenta), 50\% (purple), 30\% (blue), and 10\% (light blue) isodose curves [100\% was 55.2 Gy (RBE)].

was defined as the physical dose multiplied by the RBE of the C-ions (19). Treatment planning computed tomography (CT) and contrast-enhanced CT images were merged to precisely delineate the target. The gross tumour volume (GTV) and clinical target volume (CTV) were also delineated. CTV was obtained by adding 5-mm margins to the GTV and including the prophylactic lymph node and neuroplexus regions. The planning target volume (PTV) included the CTV with a 3-mm margin for possible positioning errors. When the PTV overlapped with an organ at risk (OAR), the margin was reduced accordingly. The PTV was calculated to be $206.7 \mathrm{~cm} 3$. The calculations for internal metals of coil embolisation were estimated using the stopping power of water because the constituent materials of the internal metals were unknown. This was done to prevent an unexpected high-dose irradiation to the downstream OAR, such as the gastrointestinal tract. The inaccuracy of this calculation was considered acceptable for safety. C-ion RT was then performed using passive irradiation. The administered dose of C-ion RT was $55.2 \mathrm{~Gy}$ (RBE) in 12 fractions for 3 weeks [4.6 Gy (RBE) per fraction], and the gating level for respiratory-gated irradiation was within $30 \%$ of the wave height around the peak exhalation. Figure 1D shows the dose distribution of the treatment plan. We created a simulated dose distribution that recalculated the treatment plan by replacing the stopping power of the internal metals from the value of water to the value of stainless steel, which is six times the stopping power of water (Figure 2E). The patient completed C-ion RT as scheduled. The study protocol was approved by the ethics committee of the Gunma University Graduate School of Medicine (HS2019-130).

Auto-activation positron emission tomography. A Clinical PET-CT scanner (Eminence STARGATE; Shimadzu Corporation, Kyoto, Japan) was used to acquire the distribution of positron emitters after $\mathrm{C}$-ion irradiation. PET-CT was performed in the sixth fraction out of 


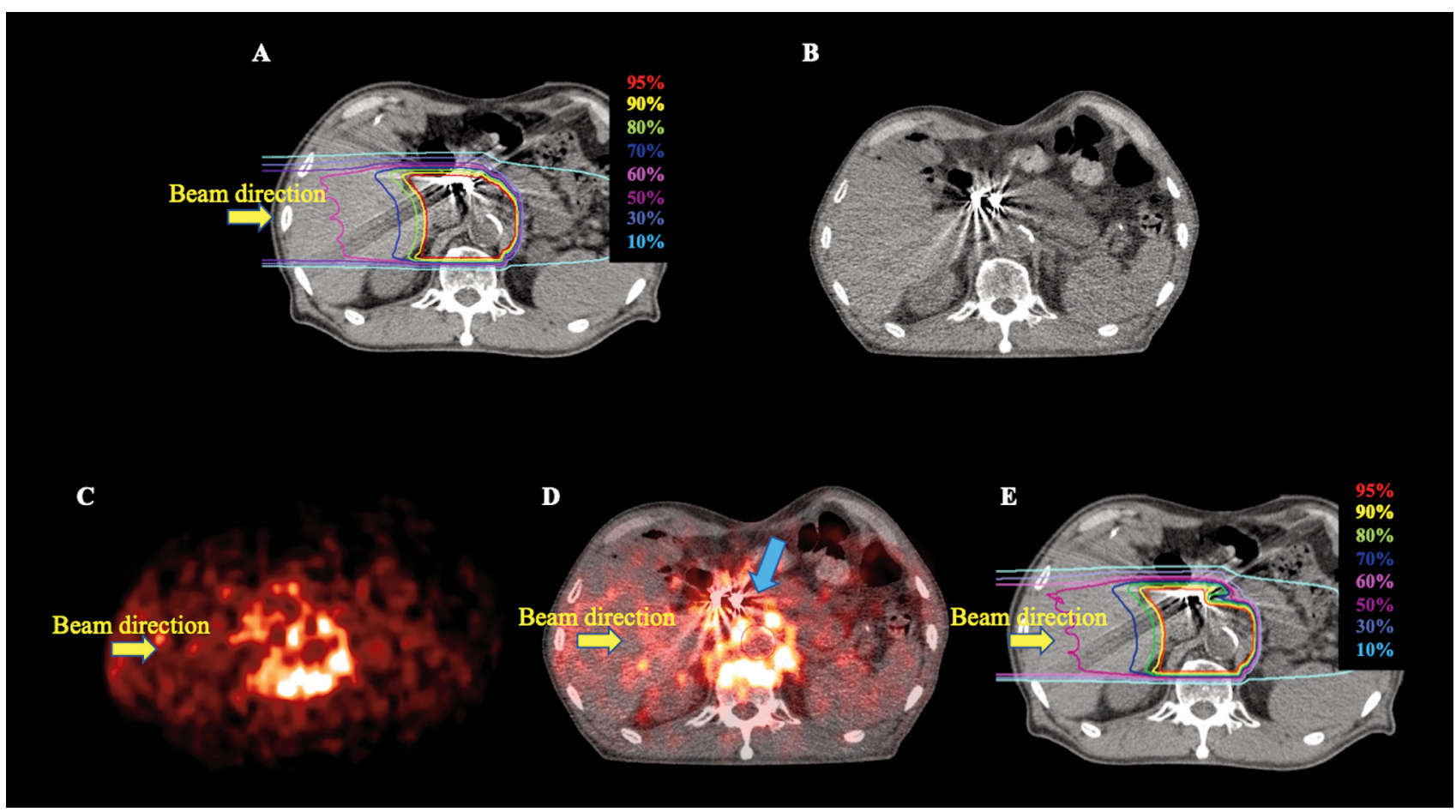

Figure 2. Dose distribution of carbon ion radiotherapy $\left(C\right.$-ion RT) and auto-activation positron emission tomography $(A A P E T)$ images with a $270^{\circ}$ beam. (A) Dose distribution of C-ion RT with $270^{\circ}$ beam in the internal metals level. (B) CT image of the internal metals level taken at the same time of PET scan. (C) AAPET image in the internal metals level. (D) Positron emitter distributions obtained by merged CT and AAPET images. Blue arrow shows low concentration of positron emitter compared to dose distribution of C-ion RT. (E) Dose distribution of C-ion $R T$ with $270^{\circ}$ beam in the internal metals level calculated using stopping power of stainless steel (density value 6) on the internal metals. Dose distribution degradation was observed in the distal side of the internal metals which consistent low concentration of positron emitter in the distal side of the internal metals in Figure 2D. The area within the blue and red outlines are the tumour and the aorta, respectively. Highlighted are the 95\% (red), 90\% (yellow), 80\% (green), $70 \%$ (deep blue), 60\% (magenta), 50\% (purple), 30\% (blue), and 10\% (light blue) isodose curves [100\% was 4.6 Gy (RBE)].

the 12 fractions of C-ion RT. This irradiation was performed with a $270^{\circ}$ horizontal beam using a Spread-out Bragg peak of $70 \mathrm{~mm}$. The irradiation time of the $\mathrm{C}$-ion beams was $148 \mathrm{~s}$. After the irradiation, the patient was immediately taken to a PET room located near the treatment room. The patient was placed in a treatment position on the PET-CT scanner without the immobilisation device. PET-CT scan was started 10 min after C-ion RT. Next, the PET images were merged with the CT images using the bone and internal metal matching technique for comparison with the dose distribution of the treatment plan. The average count per second of the positron emitters was calculated in the following areas: irradiated field (i.e., PTV excluding distal side of the internal metals, aorta, internal metals, and bone), distal side of the internal metals, aorta, internal metals, irradiated bone, and out of the irradiated field.

\section{Results}

AAPET images were acquired and merged with CT images taken at the same time (Figure 2 and Figure 3). Figure 2A and Figure $3 \mathrm{~A}$ show the dose distribution with the $270^{\circ}$ beam in the treatment planning, Figure $2 \mathrm{~B}$ and Figure $3 \mathrm{~B}$ show the CT images taken at the same time as the PET scan, Figure 2C and Figure $3 \mathrm{C}$ show the AAPET images before merging with the CT images, and Figure 2D and Figure 3D show the AAPET images after merging with the CT images. The positron emitter distribution was consistent with the areas that received a high dose of C-ion RT, excluding the distal side of the internal metals and the aorta (Figure 2D and Figure 3D). There was a lower concentration of positron emitter in the distal side of the internal metals in the pancreatic body compared to the dose distribution of the plan (Figure 2D, blue arrow). Furthermore, there was a reduction in the positron emitters in the aorta compared to the plan dose distribution (Figure 3D, green circle). Figure 2E shows a simulated dose distribution that recalculated the treatment plan using the stopping power of stainless steel and dose distribution degradation on the distal side of the internal metals. This dose distribution degradation was matched with a low concentration of the positron emitter in the distal side of the internal metals (Figure 2D). Additionally, the concentration of the positron emitter in the bone was considerably high, while 


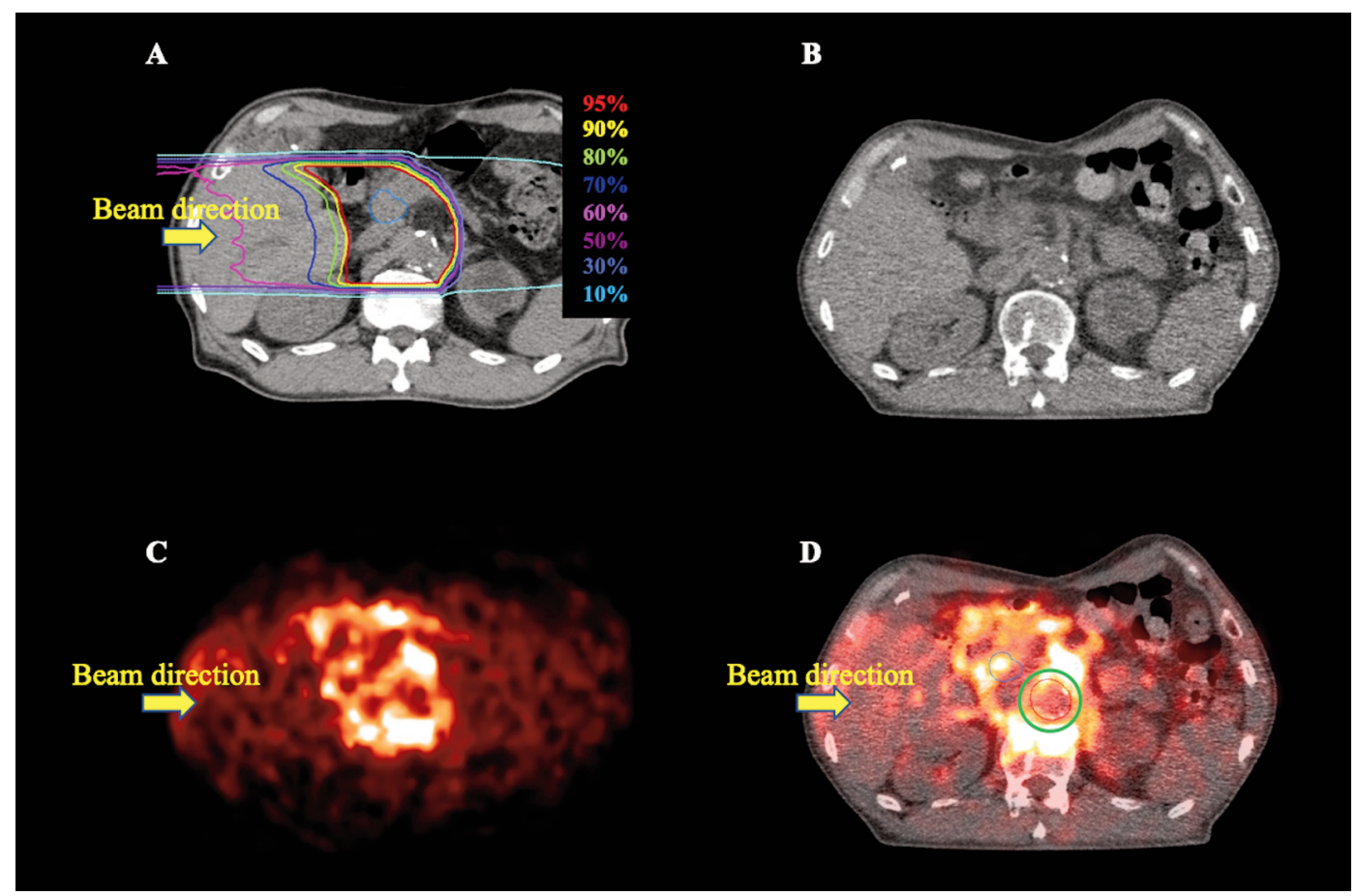

Figure 3. Dose distribution of carbon ion radiotherapy $(C$-ion $R T)$ and auto-activation positron emission tomography (AAPET) images with $270^{\circ}$ beam. (A) Dose distribution of C-ion RT with $270^{\circ}$ beam in the pancreatic head tumour level. The area within the blue outline is the tumour. (B) CT image of the pancreatic head tumour level taken at the same time of PET scan. (C) AAPET image in the pancreatic head tumour level. (D) Positron emitter distributions obtained by merged CT and AAPET images. Green circle shows a low concentration of the positron emitter compared to dose distribution of $C$-ion RT. The area within the blue and red outlines are the tumour and the aorta, respectively. Highlighted are the $95 \%$ (red), $90 \%$ (yellow), 80\% (green), 70\% (deep blue), 60\% (magenta), 50\% (purple), 30\% (blue), and 10\% (light blue) isodose curves [100\% was 4.6 Gy (RBE)].

that in the internal metal was low (Figure 2D and Figure 3D). The average count per second of the positron emitter ( \pm standard deviation) in the irradiated field, distal side of the internal metals, aorta, internal metals, irradiated bone, and out of the irradiated field were $28.3 \pm 11.6,21.5 \pm 3.7,16.2 \pm 5.1,20.1 \pm 10.5$, $49.9 \pm 15.7$, and 8.9 \pm 5.7 , respectively (Figure 4). The count per second of the positron emitter showed a relatively lower concentration in the distal side of the metals, aorta, and metals than in the irradiated field. In contrast, the positron emitter concentration was relatively higher in the irradiated bone than in the irradiated field.

\section{Discussion}

We observed a low concentration of positron emitter in the distal side of the internal metals and the aorta using AAPET, despite high-dose administration of C-ion RT at the planned target area. This may have been caused by an inaccurate calculation of the stopping power (this inaccurate calculation was considered acceptable for safety to OAR) and physiological washout. To the best of our knowledge, this is the first time such events have been visualised in a human case.
In particle therapy, specific stopping power of the materials that the beam passes, such as metals, must be calculated to create the correct dose distribution. In general, the data to convert CT values to stopping power (a CT-SP table) is prepared from measurements using ethanol and dipotassium hydrogen phosphate $(20,21)$. Therefore, the stopping power of metals with high atomic numbers cannot be calculated from the CT values. In this study, the elements of the embolisation metals were unknown, and calculations for the embolisation coils were estimated using the stopping power of the water to prevent an unexpected high-dose irradiation to the downstream OAR. When treatment planning calculations on the internal metals are performed using stainless steel, which is six times the stopping power of water, a high-dose irradiation to the downstream OAR might occur if the position of the internal metals is misaligned during actual irradiation, resulting in severe toxicities. To prevent the unexpected high-dose irradiation to the downstream OAR, beam shortening by internal metals is acceptable because of safety irradiation, and treatment planning calculations were accordingly performed using the stopping power of the water. In the current study, a low concentration of the positron emitter area compared to the 


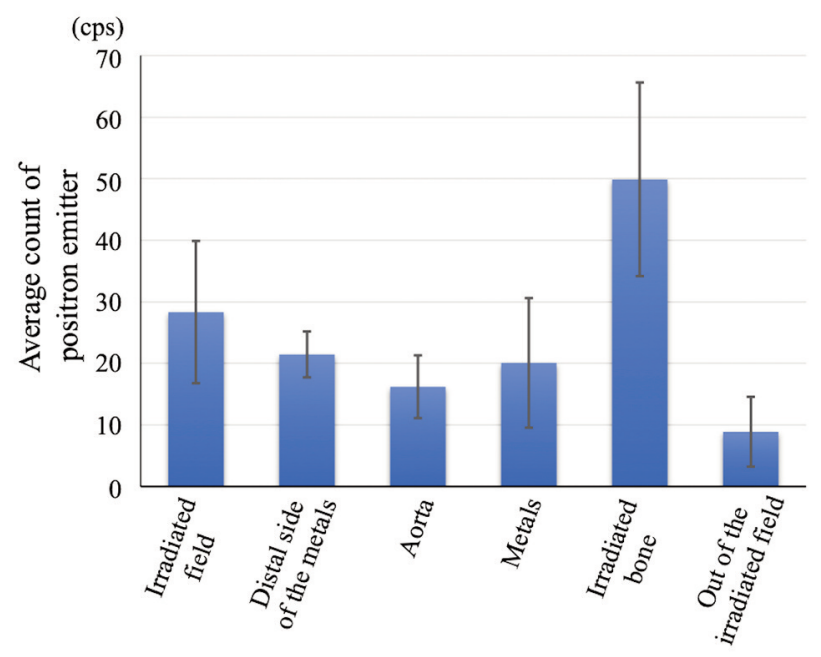

Figure 4. Data are presented as the average \pm standard deviation of positron emitter in the irradiated field, distal side of the internal metals, aorta, metals, irradiated bone, and out of the irradiated field. cps, Count per seconds.

dose distribution was observed on the distal side of the internal metals, which could be due to the incorrect dose calculations for safety. This suggests that C-ion RT was performed safely. When a beam passes through an internal metal object and an accurate dose calculation is required downstream of the metal objects, the stopping power must be determined from the constituent materials or an experimental measurement. Additionally, verifying the beam's range consistency with the treatment plan using modalities such as AAPET is recommended at the time of the first treatment when using a beam that passes through the internal metal.

Previous studies reported that positron emitters generated by $\mathrm{C}$-ion beams are transported by biofluids $(12,15-17)$. However, the transportation of activated elements in clinical cases has not yet been fully observed. Compared to the dose distribution and AAPET image in the current case, there were significantly fewer positron emitters in the aorta, which could have been transported and washed out by the blood flow. Although the PET scan was performed at the same time as the therapeutic irradiation, the radioisotope production in the blood could not be accurately measured due to its transport before the decay. In addition, in this study, there was a 10-min delay from the end of the treatment to the start of PET. Thus, it could be considered that some portion of the positron emitters generated outside the aorta were also washed out, and it is unclear from these images that the manner and pace at which positron emitters were transported.

A high signal was detected within the bones by PET, as previously reported $(11,14,22)$. Fragments of calcium and phosphorus, which are the main components of bone, are believed to be primarily responsible for this observation. In contrast, the concentration of positron emitters in the internal metal region appeared to be low. Although positron-emitting radionuclides with short half-lives are mostly light-element isotopes, coils are often made of heavy metal alloys or stainless steel, which may generate low amounts of long half-life positron emitters $\left(e . g .,{ }^{11} \mathrm{C},{ }^{13} \mathrm{~N}\right.$, and $\left.{ }^{15} \mathrm{O}\right)$ in the spallation. This could explain the low concentration of the positron emitters in the internal metal region.

In our facility, the PET equipment is in a room separate from the irradiation room because of difficulty in installation in the same room owing to its large size. Therefore, real-time monitoring of beam attenuation and transportation of positron emitters was not possible. Additionally, a quantitative analysis needs to be performed carefully, especially for comparisons between different organs. Thus, real-time monitoring of irradiation and multiple slice visualisation of positron emitter transportation constitute pertinent future prospects in examining the visualisation of C-ion RT irradiation. Currently, real-time monitoring using OpenPET and medical Compton cameras is being researched $(12,23)$.

In conclusion, we observed range shortening of C-ion beams by internal metals and washout of intravascular positron emitters compared to the dose distribution, using AAPET in a human case. These results might contribute to the future development of verification method of C-ion RT.

\section{Conflicts of Interest}

Tatsuya Ohno received research funding from Hitachi. All other Authors declare no conflicts of interest.

\section{Authors' Contributions}

Conceptualisation, S.S.; investigation, S.S. and M.S.; resources, S.S. and M.O.; patient treatment, S.S. and M.O.; writing - original draft preparation, S.S. and M.S.; writing - review and editing, S.S., M.S., M.O., and T.O.; visualisation, S.S. and M.S.; supervision, T.O.; project administration, T.O.; funding acquisition, T.O.

\section{Acknowledgements}

The Authors would like to thank their colleagues at the Gunma University Heavy Ion Medical Center and the Department of Radiation Oncology.

\section{References}

1 Shiba S, Okamoto M, Kiyohara H, Ohno T, Kaminuma T, Asao T, Ojima H, Shirabe K, Kuwano H and Nakano T: Prospective observational study of high-dose carbon-ion radiotherapy for pelvic recurrence of rectal cancer (GUNMA 0801). Front Oncol 9: 702, 2019. PMID: 31417874. DOI: 10.3389/fonc.2019.00702

2 Shiba S, Shibuya K, Okamoto M, Okazaki S, Komatsu S, Kubota Y, Nakano $\mathrm{T}$ and Ohno T: Clinical impact of Hypofractionated carbon ion radiotherapy on locally advanced hepatocellular carcinoma. Radiat Oncol 15(1): 195, 2020. PMID: 32795340. DOI: 10.1186/s13014-020-01634-z 
3 Ohno T, Noda SE, Murata K, Yoshimoto Y, Okonogi N, Ando K, Tamaki T, Kato S, Hirakawa T, Kanuma T, Minegishi T, Nakano $\mathrm{T}$ and Working Group for Gynecologic Cancer: Phase I study of carbon ion radiotherapy and image-guided brachytherapy for locally advanced cervical cancer. Cancers (Basel) 10(9): 338, 2018. PMID: 30231543. DOI: 10.3390/cancers 10090338

4 Imai R, Kamada T, Araki N and Working Group for Bone and Soft Tissue Sarcomas: Carbon ion radiation therapy for unresectable sacral chordoma: an analysis of 188 cases. Int J Radiat Oncol Biol Phys 95(1): 322-327, 2016. PMID: 27084649. DOI: $10.1016 /$ j.ijrobp.2016.02.012

5 Mohamad O, Imai R, Kamada T, Nitta Y, Araki N and Working Group for bone and soft tissue sarcoma: Carbon ion radiotherapy for inoperable pediatric osteosarcoma. Oncotarget 9(33): 2297622985, 2018. PMID: 29796166. DOI: 10.18632/oncotarget.25165

6 Kanai T, Endo M, Minohara S, Miyahara N, Koyama-ito H, Tomura H, Matsufuji N, Futami Y, Fukumura A, Hiraoka T, Furusawa Y, Ando K, Suzuki M, Soga F and Kawachi K: Biophysical characteristics of HIMAC clinical irradiation system for heavy-ion radiation therapy. Int J Radiat Oncol Biol Phys 44(1): 201-210, 1999. PMID: 10219815. DOI: 10.1016/s0360-3016(98)00544-6

7 Abe S, Kubota Y, Shibuya K, Koyama Y, Abe T, Ohno T and Nakano T: Fiducial marker matching versus vertebral body matching: Dosimetric impact of patient positioning in carbon ion radiotherapy for primary hepatic cancer. Phys Med 33: 114-120, 2017. PMID: 28057427. DOI: 10.1016/j.ejmp.2016.12.018

8 Irie D, Saitoh JI, Shirai K, Abe T, Kubota Y, Sakai M, Noda SE, Ohno $\mathrm{T}$ and Nakano $\mathrm{T}$ : Verification of dose distribution in carbon ion radiation therapy for stage I lung cancer. Int J Radiat Oncol Biol Phys 96(5): 1117-1123, 2016. PMID: 27869084. DOI: 10.1016/j.ijrobp.2016.09.002

9 Sakai M, Kubota Y, Saitoh JI, Irie D, Shirai K, Okada R, Torikoshi M, Ohno T and Nakano T: Robustness of patient positioning for interfractional error in carbon ion radiotherapy for stage I lung cancer: Bone matching versus tumor matching. Radiother Oncol 129(1): 95-100, 2018. PMID: 29100701. DOI: 10.1016/j.radonc.2017.10.003

10 Shiba S, Saitoh JI, Irie D, Shirai K, Abe T, Kubota Y, Sakai M, Okada R, Ohno T and Nakano T: Potential pitfalls of a fiducial marker-matching technique in carbon-ion radiotherapy for lung cancer. Anticancer Res 37(10): 5673-5680, 2017. PMID: 28982885. DOI: 10.21873/anticanres.12003

11 Bauer J, Unholtz D, Sommerer F, Kurz C, Haberer T, Herfarth K, Welzel T, Combs SE, Debus J and Parodi K: Implementation and initial clinical experience of offline PET/CT-based verification of scanned carbon ion treatment. Radiother Oncol 107(2): 218-226, 2013. PMID: 23647759. DOI: 10.1016/j.radonc.2013.02.018

12 Parajuli RK, Sakai M, Kada W, Torikai K, Kikuchi M, Arakawa $\mathrm{K}$, Torikoshi $\mathrm{M}$ and Nakano T: Annihilation gamma imaging for carbon ion beam range monitoring using Si/CdTe Compton camera. Phys Med Biol 64(5): 055003, 2019. PMID: 30669125. DOI: $10.1088 / 1361-6560 / a b 00 b 2$

13 Parajuli RK, Sakai M, Kada W, Torikai K, Kikuchi M, Arakawa $\mathrm{K}$, Torikoshi $\mathrm{M}$ and Nakano T: Annihilation gamma imaging for carbon ion beam range monitoring using $\mathrm{Si} / \mathrm{CdTe}$ Compton camera. Phys Med Biol 64(5): 055003, 2019. PMID: 30669125. DOI: 10.1088/1361-6560/ab00b2

14 Shimizu M, Sasaki R, Miyawaki D, Nishimura H, Demizu Y, Akagi T, Suga D, Sakamoto H, Murakami M, Sugimura K and Hishikawa Y: Physiologic reactions after proton beam therapy in patients with prostate cancer: significance of urinary autoactivation. Int J Radiat Oncol Biol Phys 75(2): 580-586, 2009. PMID: 19735884. DOI: 10.1016/j.ijrobp.2009.02.085

15 Mizuno H, Tomitani T, Kanazawa M, Kitagawa A, Pawelke J, Iseki Y, Urakabe E, Suda M, Kawano A, Iritani R, Matsushita S, Inaniwa T, Nishio T, Furukawa S, Ando K, Nakamura YK, Kanai $\mathrm{T}$ and Ishii $\mathrm{K}$ : Washout measurement of radioisotope implanted by radioactive beams in the rabbit. Phys Med Biol 48(15): 2269-2281, 2003. PMID: 12953897. DOI: 10.1088/0031-9155/48/15/302

16 Ammar C, Frey K, Bauer J, Melzig C, Chiblak S, Hildebrandt M, Unholtz D, Kurz C, Brons S, Debus J, Abdollahi A and Parodi K: Comparing the biological washout of $\beta+$-activity induced in mice brain after $12 \mathrm{C}$-ion and proton irradiation. Phys Med Biol 59(23): 7229-7244, 2014. PMID: 25383509. DOI: $10.1088 / 0031-9155 / 59 / 23 / 7229$

17 Toramatsu C, Mohammadi A, Wakizaka H, Seki C, Nishikido F, Sato S, Kanno I, Takahashi M, Karasawa K, Hirano Y and Yamaya T: Biological washout modelling for in-beam PET: rabbit brain irradiation by ${ }^{11} \mathrm{C}$ and ${ }^{15} \mathrm{O}$ ion beams. Phys Med Biol 65(10): 105011, 2020. PMID: 32235057. DOI: 10.1088/1361-6560/ab8532

18 Brierley JD, Gospodarowicz MK and Wittekind C: International Union Against Cancer (UICC): TNM Classification of Malignant Tumours. 8th ed. Oxford, Wiley-Blackwell, 2017.

19 Inaniwa T, Kanematsu N, Matsufuji N, Kanai T, Shirai T, Noda K, Tsuji H, Kamada T and Tsujii H: Reformulation of a clinicaldose system for carbon-ion radiotherapy treatment planning at the National Institute of Radiological Sciences, Japan. Phys Med Biol 60(8): 3271-3286, 2015. PMID: 25826534. DOI: 10.1088/ 0031-9155/60/8/3271

20 Kanematsu N, Matsufuji N, Kohno R, Minohara S and Kanai T: A CT calibration method based on the polybinary tissue model for radiotherapy treatment planning. Phys Med Biol 48(8): 1053-1064, 2003. PMID: 12741501. DOI: 10.1088/0031-9155/48/8/307

21 Schaffner B and Pedroni E: The precision of proton range calculations in proton radiotherapy treatment planning: experimental verification of the relation between CT-HU and proton stopping power. Phys Med Biol 43(6): 1579-1592, 1998. PMID: 9651027. DOI: 10.1088/0031-9155/43/6/016

22 Parodi K, Paganetti H, Shih HA, Michaud S, Loeffler JS, DeLaney TF, Liebsch NJ, Munzenrider JE, Fischman AJ, Knopf A and Bortfeld T: Patient study of in vivo verification of beam delivery and range, using positron emission tomography and computed tomography imaging after proton therapy. Int J Radiat Oncol Biol Phys 68(3): 920-934, 2007. PMID: 17544003. DOI: 10.1016/j.ijrobp.2007.01.063

23 Tashima H, Yoshida E, Inadama N, Nishikido F, Nakajima Y, Wakizaka H, Shinaji T, Nitta M, Kinouchi S, Suga M, Haneishi $\mathrm{H}$, Inaniwa $\mathrm{T}$ and Yamaya $\mathrm{T}$ : Development of a small single-ring OpenPET prototype with a novel transformable architecture. Phys Med Biol 61(4): 1795-1809, 2016. PMID: 26854528. DOI: $10.1088 / 0031-9155 / 61 / 4 / 1795$

Received August 18, 2021

Revised September 16, 2021

Accepted September 30, 2021 